\title{
Racial Equity in the Fight Against COVID-19: A Qualitative Study Examining the Importance of Collecting Race-Based Data in the Canadian Context
}

Ranie Ahmed ( $\nabla$ ranie.ahmed@mail.utoronto.ca )

University of Toronto https://orcid.org/0000-0002-4877-5038

Omer Jamal

University of Toronto Scarborough

Waleed Ishak

University of Toronto

Kiran Nabi

University of Toronto Scarborough

Nida Mustafa

University of Toronto Scarborough

\section{Research}

Keywords: Racial Equity, COVID-19, Black Community, Social Determinants of Health, Racialized Communities

Posted Date: January 28th, 2021

DOl: https://doi.org/10.21203/rs.3.rs-152695/v1

License: (c) (1) This work is licensed under a Creative Commons Attribution 4.0 International License.

Read Full License 


\section{Abstract \\ Background}

A failure to ensure racial equity in response to the COVID-19 pandemic has caused Black communities in Canada to disproportionately be impacted. The aim of the current study was to determine the needs and concerns of Black communities in the Greater Toronto Area (GTA) and to highlight the importance of collecting race-based COVID-19 data.

\section{Methods}

Six qualitative interviews were conducted with local community health centre leaders who serve a high population of racialized communities within the GTA. Content analysis was used to extract the main themes and concerns raised during the interviews.

\section{Results}

The findings from this study provide further evidence of the disproportionate impact COVID-19 has had on Black and other racialized communities. Difficulty self-isolating due to overcrowded housing, food insecurity, and less social support for seniors were concerns identified by community health leaders. Also, enhanced financial support for front-line workers, such as Personal Support Workers (PSWs), was an important concern raised. In order to lessen the impact of the pandemic on these communities, leaders noted the need for greater accessibility of testing centres in these areas and a greater investment in tailored health promotion approaches.

\section{Conclusions}

Overall, our findings point to the importance of collecting race-based data to ensure an equitable response to the pandemic. The current "one size fits all" response is not effective for all individuals, especially Black communities. Not all populations have access to the same resources, nor do they live in the same conditions [1]. A deeper consideration of the social determinants of health are needed when implementing COVID-19 policies and responses. Also, a lack of attention to Black communities only continues to perpetuate the underestimated issue of anti-black racism prevalent in Canada.

\section{Background}

COVID-19 is an infectious disease caused by the SARS-CoV-2 virus, targeting the respiratory and gastrointestinal tract of humans [2]. It is spread through respiratory droplets and airborne transmission, and manifests both asymptomatically and symptomatically within individuals [3]. Good hygiene, 
including hand sanitization, the use of facial masks/coverings and social distancing, are key protective measures identified by public health organizations, agencies and leaders across the globe.

Although the virus does not discriminate in terms who it infects, research has shown that marginalized communities are at greater risks due to an inequitable distribution of resources, such as lack of government funding, housing, social support, and food insecurity [1]. Many marginalized populations are not able to adopt adequate preventative measures due to these conditions, which further increases their susceptibility to contract the virus [1]. In Canada, research shows that Black communities, in particular, are more likely to become sick and be hospitalized with COVID-19 compared to other ethnic groups [4]. For example, from May 20 to July 16 (2020), it was found that Black individuals made up $21 \%$ of the COVID-19 cases in Toronto (one of the most populated cities in the country), despite only making up 9\% of the population [4]. These communities are also disproportionately affected by certain chronic conditions, such as HIV, diabetes, food insecurity, low-income, and unstable housing in Canada [5]. The effects of COVID-19 have, therefore, further exacerbated these already present health disparities.

Although some provinces in Canada, including Ontario, have started collecting race-based data (population health outcomes stratified by racial groups; [6], earlier measures should have been taken to ensure vulnerable communities are protected during this time. It has been suggested that the province should have gathered neighborhood based COVID-19 data in the early stages of the pandemic, or even prior, to identify communities that would be most impacted [6]. This data could have guided and informed equitable response efforts, such as providing additional funding and safety resources (e.g. face masks, sanitizers) to those communities. In times of crisis, the inequitable experiences of marginalized populations need to be identified and addressed at the beginning, as these groups disproportionately bear a greater burden of suffering [7]. Hence, the goal of this project was to demonstrate the importance of collecting race-based COVID-19 data to ensure an equitable response to the current pandemic, in the Canadian context.

\section{Materials And Methods}

\section{Qualitative Study Design}

Qualitative research is a process that involves collecting and analyzing non-numerical data to achieve an improved understanding of a specific concept, opinion, or experience [8]. It provides researchers with personal accounts and experiences on various topics, and a deeper understanding of a problem to help generate new ideas for research. Unlike quantitative research, which uses statistics and numerical data, qualitative research allows researchers to further understand population health outcomes based on conversations, narratives, and discussions with those who have lived experiences of an issue, or those who work closely with members within a community.

The issue of racial equity cannot solely be examined through health statistics because in order to tackle the root causes of this issue, the lived experiences of target populations through community-based 
research is required. For example, in a study conducted in Southeast England on the accessibility of mental health services among Black and minority (BME) ethnic communities, a qualitative approach was taken by researchers [9]. This approach was taken to equitably inform the development of culturally acceptable services to improve population health. Therefore, an important perspective to understand racial equity issues is the lived experience of people who are impacted. This provides a powerful means to examine concerns and challenges affecting individuals on a daily basis, which may not be captured through traditional quantitative measures. Therefore, our project utilized a qualitative approach to better understand the needs of Black communities in the GTA.

\section{Data collection method}

After obtaining ethical approval from a University's Research Ethics Board, six qualitative interviews were conducted (over the phone or in podcast style) with community health centre leaders across the GTA between July 2020 to August 2020. The podcasts were conducted through zoom, an online communication platform, due to COVID-19 policies and guidelines.

Each interview was between 45 minutes to one hour. Community leaders were asked about issues and concerns within their communities in regards to the pandemic; for example, "what resources are currently needed that the community does not have access to?", "how important is it that our leaders understand that a one-size fits all approach is not an effective solution this pandemic?". Also, community leaders were asked for their thoughts on the importance and need for collecting race-based data in the GTA.

Once interviews were complete, they were transcribed verbatim, followed by the extraction of main themes through the use of Content Analysis [10]. This systematic method identifies important and emerging thematic findings that relate to the overall research focus/question.

\section{Participants}

Five executive directors of community health centres and one university professor took part in our study. Their expertise spanned in a broad range of disciplines, namely public health, public policy, healthcare management, and community organizing and development.

\section{Results}

The findings below identify the main concerns which arose among Black communities during the pandemic. In particular, community leaders discussed the importance of mobile testing and the role of working conditions in spreading the virus among marginalized groups. They also identified the role of anti-black racism in pandemic response and the importance of equitable health policies. Future lessons/approaches the government should take to implement equitable public health measures that are tailored to the lived experiences of Black communities, were also highlighted.

\section{Common concerns of the community}


A common concern emerging from the interviews was a reluctance among individuals from Black communities to get tested for the virus due to misunderstandings of how painful the test would be. Also, there was a fear of increased contact with individuals who have tested positive at the testing centre. This made individuals from these communities less likely to get tested for the virus. In addition, individuals were hesitant to get tested due to the possibility of a positive test, leading them to worry about taking time off work. This would mean they would need to find other sources of income to support their families (i.e. to pay rent, buy groceries).

Many members of these communities also lost jobs and were not eligible for the Canadian Emergency Response Benefit (CERB). This led to other concerns such as the affordability of food and personal protective equipment (PPE). As many members from these communities have a lower socio-economic status, the loss of employment further exacerbated income inequality.

These concerns provide clarity on the negative effects of COVID-19 on Black communities, and the impact of this on the communities' physical and mental health.

\section{Mobile testing}

Mobile testing became available in non-racialized GTA neighborhoods (which were socially less burdened) weeks before regions that were most affected by COVID-19. In particular, those communities with inadequate housing and poor access to healthcare were delayed in receiving such resources. Communities with greater populations of racialized individuals suffered the most during the pandemic because there was no prior knowledge of the needs of these communities or what health concerns were already present. Community leaders suggested that differences in resource allocation between certain communities points to systemic discrimination, neglect, and a lack of prioritization of racialized communities which are in need the most.

\section{Working conditions}

A disproportionate number of Black female personal support workers (PSW) were employed in nursing homes which were most affected during the pandemic. This increased their chances of contracting the virus themselves, as well as becoming carriers for their family members.

Also, community leaders indicated a correlation between low-income jobs and the risk of contracting COVID-19, in these communities specifically. As one leader highlighted, "a lot of racialized and marginalized communities live in low-income conditions, where their employment situation is precarious to an extent where they aren't able to access financial assistance" (Executive Director, Community Health Centre in Scarborough, Toronto). Thus, to make ends meet, some Black individuals have to work multiple jobs to receive adequate income.

\section{Psychological Effects}

The psychological effects of COVID-19 are expected to have a significant impact on populations across the country, especially those of marginalized communities who face a greater burden. For example, many 
individuals from these regions must work throughout the pandemic as they do not have the option of staying home. Often times, these individuals are living with vulnerable family members (i.e. seniors; those with chronic conditions), and therefore, have to send them to other member's houses to reduce the spread of COVID-19. This adds to the isolation, loneliness, and depression these communities face. The psychological effects are cumulating; therefore, resources need to be tailored and made accessible in these communities. One community leader noted that, "there are accessibility issues to mental health support in these communities already" (Executive Director, Community Health Centre in Scarborough, Toronto). Thus, efforts need to be made to increase access to mental health services in Black communities.

\section{Anti-black racism}

Community leaders highlighted that earlier measures should have been implemented to avoid the high percentage of COVID-19 cases among Black people and other people of colour. Racial inequities faced by communities in the healthcare system continues to be a long-standing concern even prior to the current pandemic. Health officials, therefore, need to prioritize the health of minority populations through the implementation of tangible policies that will produce meaningful change.

\section{Policies}

It was also noted that health officials need to review policies and create new ways to response to COVID19 , which target anti-black racism, specifically. Similar to the importance of collecting race-based data, it is important for policies to reflect the lived experiences of racialized populations in a real, structural, and meaningful way. Community leaders identified that many institutions already have such policies in place; however, these policies do not always translate into practice.

\section{Lessons/Future Approaches}

The provincial delay to collect race-based data is an important lesson to keep in mind, according to community leaders. Black and other racialized communities are impacted by COVID-19 and (other chronic conditions) significantly more than other populations. The inequitable disparities that already exist in these communities (in terms of housing, income, food security), perpetuate these problems even further.

As one leader notes, "the common denominator is anti-black racism and a system that is generating all of these problems. With the COVID-19 pandemic and what has transpired after the Black Lives Matter movement, we as a society have been given a good opportunity to think differently about race and health, and to bring about change" (Executive Director, Community Health Centre in Scarborough, Toronto). Public health officials, governments, and the overall population, can, and should shift the needle towards equity and fairness in health, and overall quality of life.

An important point raised by those in the study was the importance of considering how different communities will access health services when the virus vaccine is ready to distribute, in order to ensure effective usage. Making vaccinations available where community members are most comfortable visiting will be important to consider. Public health officials also need to raise awareness within these 
communities and promote community engagement to overcome misinformation and myths about vaccinations. In addition, Black communities particularly have a difficult time trusting the health system, since health services, including clinical trials and vaccines, have previously been used to further racialize, marginalize, and kill members of the Black community. Therefore, community leaders encourage and support an equitable deployment plan for vaccination.

Also, community leaders mentioned that they would like to see relationship and community building within Black communities to encourage the use of testing and mobile testing units. This will ensure trust is built and members of the community feel comfortable taking the vaccination. Normally, vaccinations are given through institutions and hospitals; however, this may not be accessible to all Black community members. Therefore, there needs to be a shift in planning to ensure a more equitable approach to intervention and prevention.

\section{Discussion}

The Canadian federal, provincial, and municipal government's general response to the pandemic has been commendable, as we saw a flattening of COVID-19 cases early on. Unfortunately, there still remains gaps in the lack of community-specific responses that are racially and socially equitable. Not all individuals should be treated the same way, as people do not have the same opportunities, privileges, and access to health care [1]. More community-specific responses could have, and should be implemented, which can lead to a significant reduction in the virus cases we see today. There should be specific plans and strategies put in place for Black communities, as well as other minority groups such as Indigenous peoples and those living in low-income communities, which ensure better health outcomes for all.

In June 2020, the City of Toronto declared anti-black racism a public health crisis [11]. The question remains on what our governmental systems and structures will do to translate this declaration into the pandemic response. Public health officials need to prepare and implement an equitable response, especially knowing that $80 \%$ of COVID-19 cases are among Black people and those of colour [4].

The collection of race-based data across the country is, therefore, extremely necessary to ensure this response is equitable. Currently, public health officials have begun collecting this data, however, they need to be better prepared to desegregate the data to identify which regions are most disproportionately impacted. If action is taken now, there is promise for equitable changes within our health and social systems.

\section{Conclusion}

The COVID-19 pandemic has and continues to disproportionately impact Black communities, which highlights the importance of collecting race-based data to ensure an equitable response. The collection of this data will inform health officials on the needs of vulnerable communities, followed by effective 
resource allocation. Collecting race-based data is one step towards addressing the health inequities faced by Black communities in Canada.

\section{Abbreviations}

GTA: Greater Toronto Area, COVID- 19: Coronavirus disease 2019, PSWs: Personal Support Workers, SARS-CoV-2: Severe acute respiratory syndrome, coronavirus 2, BME: Black and minority ethnic communities, CERB: Canadian Emergency Response Benefit, PPE: Personal protective equipment

\section{Declarations}

\section{Ethics approval and consent to participate}

The research was approved by the University of Toronto's Research Ethics Board. Written consent was obtained from all study participants.

\section{Consent for publication}

Not Applicable

\section{Availability of data and materials}

The data that support the findings of this study are available at https://www.covid19racialequity.com/.

\section{Competing interests}

The authors declare that they have no competing interests

\section{Funding}

This research was supported by the University of Toronto's COVID-19 Student Engagement Award (2020).

\section{Authors' contributions}

$\mathrm{RA}, \mathrm{OJ}, \mathrm{KN}$, and WI were all involved in data collection, analysis, and write-up. NM was the senior supervisor of the project.

\section{Acknowledgements}

Not Applicable

\section{References}

1. Kantamneni N. The impact of the COVID-19 pandemic on marginalized populations in the United States: A research agenda. Journal of Vocational Behavior, 119, 103439. 2020; 
doi:10.1016/j.jvb.2020.103439.

2. Li T. Diagnosis and clinical management of severe acute respiratory syndrome Coronavirus 2 (SARSCoV-2) infection: An operational recommendation of Peking Union Medical College Hospital (V2.0). Emerging Microbes \& Infections, 9(1), 582-585. 2020; doi:10.1080/22221751.2020.1735265.

3. Helmy Y. A, Fawzy M, Elaswad A, Sobieh A, Kenney S. P, \& Shehata A. A. The COVID-19 Pandemic: A Comprehensive Review of Taxonomy, Genetics, Epidemiology, Diagnosis, Treatment, and Control. Journal of Clinical Medicine, 9(4), 1225. 2020. doi:10.3390/jcm9041225.

4. Cheung J. Black people and other people of colour make up $83 \%$ of reported COVID-19 cases in Toronto. CBC 2020. https://www.cbc.ca/news/canada/toronto/toronto-covid-19-data-1.5669091. Accessed 20 Aug 2020.

5. Gardezi F, Calzavara L, Husbands W, et al. Experiences of and responses to HIV among African and Carribean communities in Toronto. AIDS Care. 2008; doi: 1080/09540120701693966.

6. Rizvic S. Why race-based data matters in health care: Institute for Canadian Citizenship: Institut pour la citoyenneté canadienne. 2020. https://www.inclusion.ca/article/why-race-based-data-matters-inhealth-care/. Accessed 06 December 2020.

7. Garg S, Kim L, Whitaker M, et al. Hospitalization Rates and Characteristics of Patients Hospitalized with Laboratory-Confirmed Coronavirus Disease 2019 - COVID-NET, 14 States, March 1-30, 2020. MMWR Morb Mortal Wkly Rep 2020; 69:458-464. 10.15585/mmwr.mm6915e3.

8. Aspers P, Corte U. What is Qualitative in Qualitative Research. Qualitative Sociology. 2019; doi: 10.1007/s11133-019-9413-7

9. Memon A, Taylor K, Mohebati L. M, Sundin J, Cooper M, Scanlon T, \& Visser R. D. Perceived barriers to accessing mental health services among black and minority ethnic (BME) communities: A qualitative study in Southeast England. BMJ Open, 6(11). 2016; doi:10.1136/bmjopen-2016-012337.

10. Flick, U. Mapping the Field. In the SAGE handbook qualitative data analysis. Los Angeles: SAGE; 2014. (pp. 3-19).

11. Boisvert N. Toronto Board of Health declares anti-Black racism a public health crisis. CBC News. 2020. https://www.cbc.ca/news/canada/toronto/board-of-health-anti-black-racism-1.5603383. Accessed 25 November 2020. 